\title{
Spin noise signatures of the self-induced Larmor precession
}

\author{
I. I. Ryzhov $\odot{ }^{1,2}$ V. O. Kozlov $\odot,{ }^{1,2}$ N. S. Kuznetsov $\odot,{ }^{1}$ I. Yu. Chestnov $\odot,{ }^{3,4,5}$ A. V. Kavokin, ${ }^{3,4,6,7}$ A. Tzimis, ${ }^{8,9}$ \\ Z. Hatzopoulos, ${ }^{8}$ P. G. Savvidis $\odot,{ }^{3,4,8,9,10}$ G. G. Kozlov, ${ }^{2}$ and V. S. Zapasskii ${ }^{2}$ \\ ${ }^{1}$ Photonics Department, St. Petersburg State University, Peterhof, 198504 St. Petersburg, Russia \\ ${ }^{2}$ Spin Optics Laboratory, St. Petersburg State University, Peterhof, 198504 St. Petersburg, Russia \\ ${ }^{3}$ Westlake University, School of Science, 18 Shilongshan Road, Hangzhou 310024, Zhejiang Province, China \\ ${ }^{4}$ Westlake Institute for Advanced Study, Institute of Natural Sciences, 18 Shilongshan Road, Hangzhou 310024, Zhejiang Province, China \\ ${ }^{5}$ Vladimir State University, 600000 Vladimir, Russia \\ ${ }^{6}$ Spin Optics Laboratory, St. Petersburg State University, St. Petersburg 198504, Russia \\ ${ }^{7}$ Russian Quantum Centre, 100 Novaya Street, 143025 Skolkovo, Moscow Region, Russia \\ ${ }^{8}$ Foundation for Research and Technology-Hellas, Institute of Electronic Structure and Laser, P.O. Box 1527, Heraklion, Crete 71110, Greece \\ ${ }^{9}$ Department of Materials Science and Technology, University of Crete, P.O. Box 2208, Heraklion, Crete 71003, Greece \\ ${ }^{10}$ Department of Nanophotonics and Metamaterials, ITMO University, St. Petersburg, 197101, Russia
}

(Received 31 March 2020; revised manuscript received 1 June 2020; accepted 3 June 2020; published 18 June 2020)

\begin{abstract}
Bose-Einstein condensates of exciton-polaritons are known for their fascinating coherent and polarization properties. The spin state of the condensate is reflected in polarization of the exciton-polariton emission, with temporal fluctuations of this polarization being, in general, capable of reflecting quantum statistics of polaritons in the condensate. To study the polarization properties of optically trapped polariton condensates, we take advantage of the spin noise spectroscopy technique. The ratio between the noise of ellipticity of the condensate emission and its polarization plane rotation noise is found to be dependent, in a nontrivial way, on the intensity of continuous wave nonresonant laser pumping. We show that the interplay between the ellipticity and the rotation noise can be explained in terms of the competition between the self-induced Larmor precession of the condensate pseudospin and the static polarization anisotropy of the microcavity.
\end{abstract}

DOI: 10.1103/PhysRevResearch.2.022064

Introduction. The bosonic condensates of excitonpolaritons are unique physical objects exhibiting spontaneous coherence in hybrid driven-dissipative systems. One of their most fascinating properties is an ability to emit a coherent light in a way typical of conventional lasers but with no need of any population inversion [1]. The exciton-polaritons arise due to the strong coupling between the semiconductor excitons and the quantized light [2]. Therefore the macroscopic coherent state of the polariton condensate inherits the spin degree of freedom from both polariton constituents, that is correlated with the polarization of the light emitted by the condensate. In particular, polaritons formed by the coupling of the heavy hole excitons with photons have two possible spin projections on the structure growth axis (either +1 or -1 ) which correspond to the right and left circular polarizations of the emitted photons [3].

Although the exciton-polaritons have been detected in a wide variety of systems [4-8] including Fabry-Perot microcavities [9-11] and planar waveguides [12], the most thoroughly investigated system is a semiconductor microcavity.

Published by the American Physical Society under the terms of the Creative Commons Attribution 4.0 International license. Further distribution of this work must maintain attribution to the author(s) and the published article's title, journal citation, and DOI.
Polaritons can be excited optically both resonantly and nonresonantly $[13,14]$, as well as by an electric current even at room temperature $[4,11,15]$.

Despite the well developed theoretical basis of excitonpolariton emitters, there are still a number of experimentally unrevealed aspects of their microscopic dynamics. In particular, the effect of self-induced Larmor precession, well known theoretically $[16,17]$ and studied in pulsed and continuous wave (CW) resonant [18] experiments, was never observed under nonresonant $\mathrm{CW}$ excitation of the condensate. This effect consists of the precession of the Stokes vector of the condensate of exciton-polaritons about an effective magnetic field induced by the polariton self-interaction within the condensate and oriented along the structure growth axis. Being a peculiar manifestation of the anisotropy of the exchange interaction between excitons in semiconductor quantum wells (QWs), the effect of self-induced Larmor precession is responsible for the fast polarization dynamics [18] of the resonantly excited polariton condensate and for the polarization depinning effect $[19,20]$ observed in the pulsed regime of nonresonant excitation. However, under CW nonresonant pumping conditions, most of the experimental techniques fail to reveal the presence of the self-induced Larmor precession effect since they provide information on time-integrated or time-averaged polarization signals. In this context, the spin noise spectroscopy (SNS) technique, widely used in atomic and solid state systems for spin dynamics investigation 


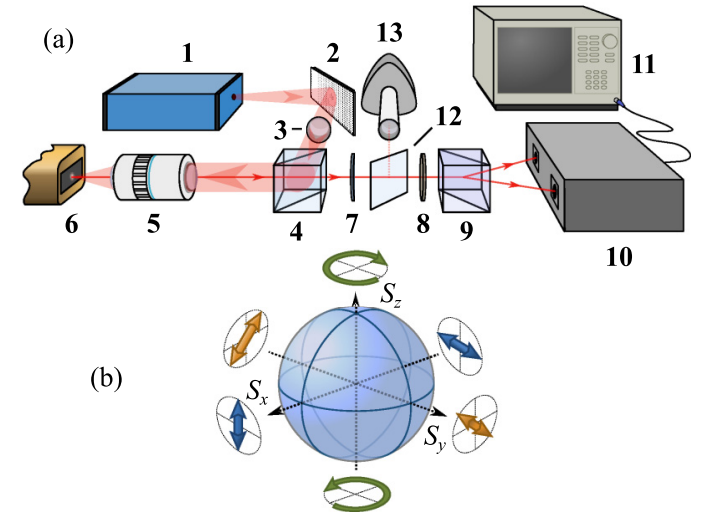

FIG. 1. (a) Schematics of the experimental setup. The description is given in the text. (b) The Poincare sphere with poles corresponding to left- and right-circular polarized light and equator corresponding to various orientations of the polarization plane of a linear polarized light.

[21-23], can be useful. It was found to be extremely helpful in exciton-polariton condensate studies [24], being informative even in the case when the information seems to be totally lost [25].

In this paper, we employ SNS to study the polarization noise properties of the optically trapped polariton condensate. Quite surprisingly, even at almost fully nonpolarized condensate emission, the behavior of the emission noise demonstrates a strong asymmetry between the ellipticity noise and the polarization plane azimuth noise. We attribute the observed asymmetry to the presence of the self-induced Larmor precession of polarization of the continuously pumped polariton condensate. This observation paves the way towards application of semiconductor microcavities in optical spintronic devices as proposed in [26].

Experimental setup. To be specific, we study the polarization properties of the polariton condensate formed in an ultrahigh-finesse $(Q \approx 31000$ corresponding to the polariton lifetime $\tau_{p} \approx 100 \mathrm{ps)} 5 \lambda / 2$ microcavity. The cavity is formed by an $\mathrm{Al}_{0.3} \mathrm{Ga}_{0.7} \mathrm{As}$ gap with four sets of three 12-nm GaAs QWs located at the antinodes of the cavity mode. The top (bottom) Bragg mirror is composed of 45 (50) $\mathrm{AlAs} / \mathrm{Al}_{0.15} \mathrm{Ga}_{0.85}$ As layers.

The polariton condensate is excited by the nonresonant pump of continuous-wave Ti:sapphire (TD Scan) laser; see Fig. 1 (label 1). The wavelength of the pump was chosen to match the first next-to-stop-band reflectivity dip at $\sim 766$ nm. The pump laser emission was directed to a Full HD microelectromechanical mirror system $(1920 \times 1080$ pixels DLP) (2) which serves for the manipulation of the pump beam spatial profile. The reflected image was collimated by a $15 \mathrm{~cm}$ lens (3) and transmitted through a nonpolarizing beam splitter (4) and 50× microobjective (5) to the sample (6) that was placed in a closed-cycle cold-finger cryostat (Montana Cryostation). The emitted light passed in an autocollimation geometry through an optical low-pass filter (7) to a conventional spin noise detection setup [22,24], which consists of a quarter-wave plate (8), a polarizing beam splitter (9), the balanced photoreceiver (10), and the spectrum analyzer (Tektronix RSA5103) (11). The real-space distribution of the condensate emission was sampled by a nonpolarizing plate (12) and the infrared camera (Andor Luca R-604) (13).

Polarization measurements require a special care about the spatial and spectral homogeneity of the condensate state. An undesirable fragmentation of the condensate suppresses the polarization signal collected from the whole condensate. In our case, a high degree of homogeneity of the QW interface along with the long polariton lifetime allows for the trapping of the condensate with pure shape. Applying the specific spatial profile of the nonresonant pump spot (see the insets in Fig. 2) with the DLP modulator we confine polaritons in an optical trap. The pump creates a cloud of incoherent excitons [27] which push polaritons to the nonexcited region due to the exciton repulsion. The polariton confinement leads to the formation of a more pure condensate state than in the case of a Gaussian pump spot profile.

Results. The measurements of the polarization degree of the polariton emission (PE) have shown that the condensate was essentially unpolarized at most of the applied pump beam profiles. The control of the mutual orientation of the quarter-wave plate (8) fast axis and the polarization beam splitter (9) main axis (characterized by angle $\theta$ ) allows one to switch between the detection of the polarization plane azimuth rotation noise, which we address hereafter as the rotation noise $(\mathrm{RN})$, and the ellipticity noise $(\mathrm{EN})$, at $\theta=0$ and $\theta=45^{\circ}$, respectively [24].

The measured polarization noise parameters can be related to the Stokes parameters of the polariton condensate emission. The latter, in turn, directly correlates with the condensate pseudospin vector $\mathbf{S}=\frac{1}{2}\left(\boldsymbol{\Psi}^{\dagger} \boldsymbol{\sigma} \boldsymbol{\Psi}\right)$, where $\sigma_{x, y, z}$ are the Pauli matrices and $\Psi=\left(\Psi_{+}, \Psi_{-}\right)^{\mathrm{T}}$ is the condensate order parameter whose components define two possible projections of the polariton spin on the structure growth axis; see Fig. 1(b). In particular, we take the horizontal and the vertical Stokes polarization (polariton pseudospin) axes coincident with the Cartesian axes of the polarization beam splitter. Then, at $\theta=$ 0 the spin noise detection scheme tracks the noise dynamics of the $S_{x}$ component of the polariton pseudospin. Likewise, if the quarter-wave plate is tilted to $\theta=45^{\circ}$, the circularly polarized component of emission is converted to the linear polarization, and the balanced scheme detects the intensity variations of circularly polarized components of the emitted light, i.e., the fluctuations of the $S_{z}$ parameter.

Fixing the shape of the pump spot, we performed several tens of pump intensity scans for both $\theta=0^{\circ}$ and $\theta=45^{\circ}$ quarter-wave plate positions. For each value of the pump intensity we collected the integral of the balanced photodiode current noise power in the frequency range of $[0,1] \mathrm{MHz}$.

Although the obtained dependencies (see Fig. 2) at high pump powers substantially vary for different excitation beam profiles, in a vast majority of cases, especially for the highly symmetrical pumps, the noise behavior around the polariton laser threshold point follows the same typical scenario. In particular, the rotation noise dominates over the noise of ellipticity at the weak pump, prevalently below lasing threshold (see the insets of Fig. 2). With the increase of the pump intensity, the noise signals of both types progressively grow. However, the EN power growth rate typically exceeds the increment of the RN power. As a result, at some point above 

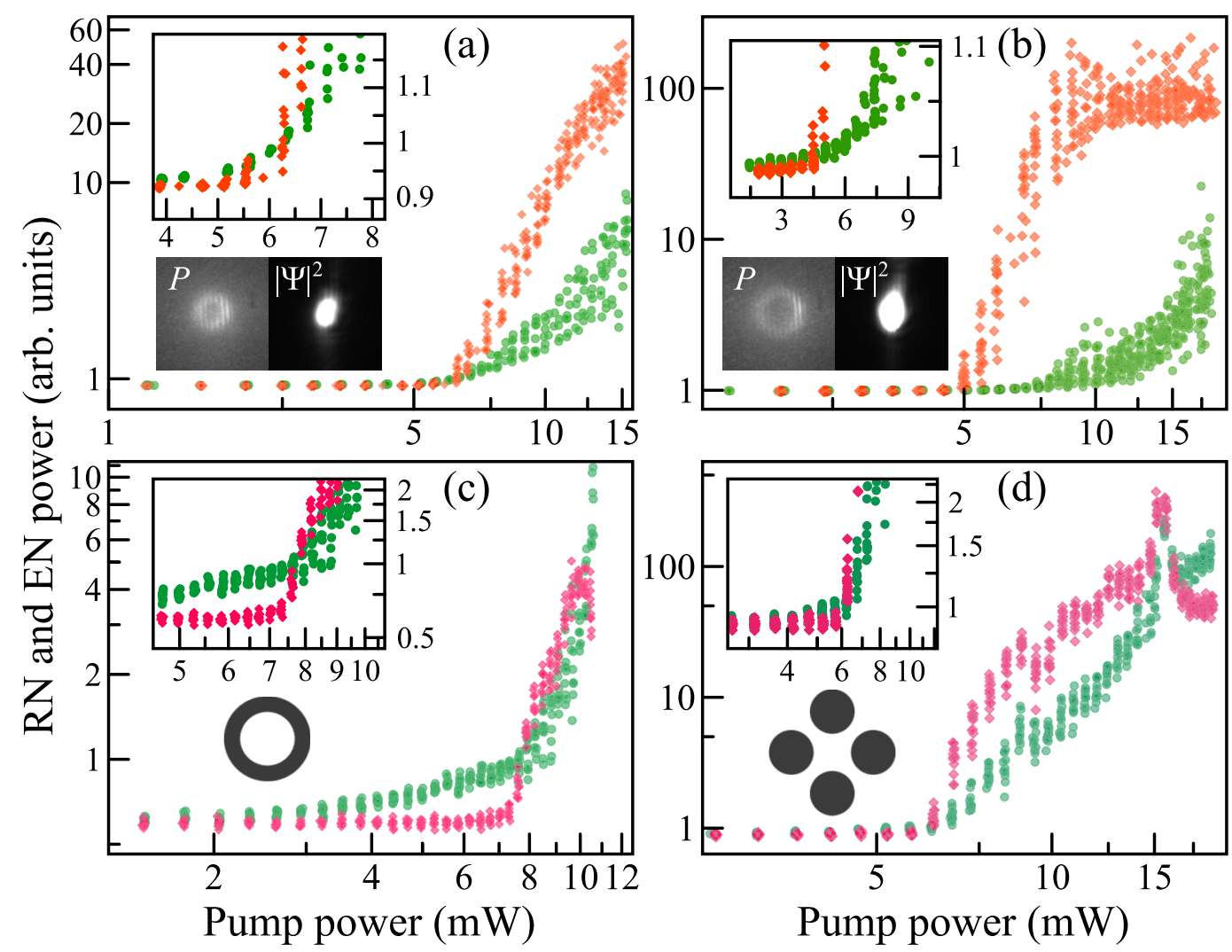

FIG. 2. The typical experimental dependencies of the rotation (green circles) and ellipticity (red rhombi) noises of the exciton-polariton condensate emission versus pump power. Panels (a)-(d) correspond to different excitation patterns. The pump beam profiles are illustrated by real-space images (top row) or by DLP patterns used (bottom row). The insets show magnified images of the intercrossing part of the dependencies discussed in the text.

the polariton lasing threshold the EN signal becomes greater than the RN. The complex behavior of the curves in the high power limit can arise from the multistability of the trapped condensate state [28] and/or from the loss of strong coupling and the threshold to photon lasing [24]. Here we focus on the behavior of RN and EN signals at low and intermediate pump power, where the system can be described in a single-mode approximation.

Discussion. We interpret the observed behavior of the condensate polarization noise in terms of the interplay between the static polarization anisotropy, which tends to suppress the circular polarization, and the spin-dependent polaritonpolariton interactions, which trigger the self-induced Larmor precession of the condensate pseudospin. We describe the state of the fluctuating order parameter of the polariton condensate in the presence of the non-resonant pump by the stochastic driven-dissipative Gross-Pitaevskii equation [19]:

$$
\begin{aligned}
i \hbar \partial_{t} \Psi_{ \pm}= & \left(\alpha_{1}\left|\Psi_{ \pm}\right|^{2}+\alpha_{2}\left|\Psi_{\mp}\right|^{2}+\alpha_{r} n\right) \Psi_{ \pm} \\
& +\frac{i \hbar}{2}\left(R n-\gamma_{c}\right) \Psi_{ \pm}+\frac{\delta_{l}}{2} \Psi_{\mp}+\sqrt{D} \eta_{ \pm}(t),
\end{aligned}
$$

for the two-component complex order parameter $\Psi=$ $\left(\Psi_{+}, \Psi_{-}\right)^{\mathrm{T}}$, whose indices correspond to polariton \pm 1 spin states. Coefficients $\alpha_{1}, \alpha_{2}$ define the interaction strengths between polaritons with the same and the opposite spins, respectively, while $\alpha_{r}$ stands for the interaction of the polaritons from the condensate with the incoherent excitons created by the nonresonant pump; $\gamma_{c}$ is the polariton lifetime. The quantity $\delta_{l}$ in the next-to-last term describes the splitting of the linear polarizations that stems from the optical anisotropy of the microcavity and, in our case, is ascribed to the inevitable mechanical strain of the structure due to the lattice disregistry of the Bragg mirror layers.

The random fluctuations of the order parameter are accounted for by the last term in Eq. (1). Taking into account the high homogeneity of the sample microcavity structure, we attribute the main source of these fluctuations to the scattering from the incoherent reservoir to the condensate state, assuming that every condensate realization is single mode. Each act of the scattering perturbs the phase of both spin components of the condensate, thus randomizing its polarization. We simulate $\eta_{ \pm}(t)$ as a Wiener process with the spectral density $D=\left\langle\eta_{i}(t) \eta_{j}^{*}\left(t^{\prime}\right)\right\rangle=\frac{1}{2} R n \delta\left(t-t^{\prime}\right) \delta_{i j}$ (see [19]), where $(i, j)=(+,-), n$ corresponds to the incoherent exciton density, and $R$ is the scattering rate. Thus the magnitude of the order parameter fluctuations appears to be dependent on the pump power insofar as the noncondensed exciton population depends on it.

Since the pumping laser frequency was tuned far above the exciton reservoir, we assume the loss of the memory of the pumping laser polarization, considering the spin-unpolarized reservoir, whose integrated density $n$ is governed by the rate equation

$$
\partial_{t} n=P-\gamma_{r} n-R n\left(\left|\Psi_{+}\right|^{2}+\left|\Psi_{-}\right|^{2}\right),
$$



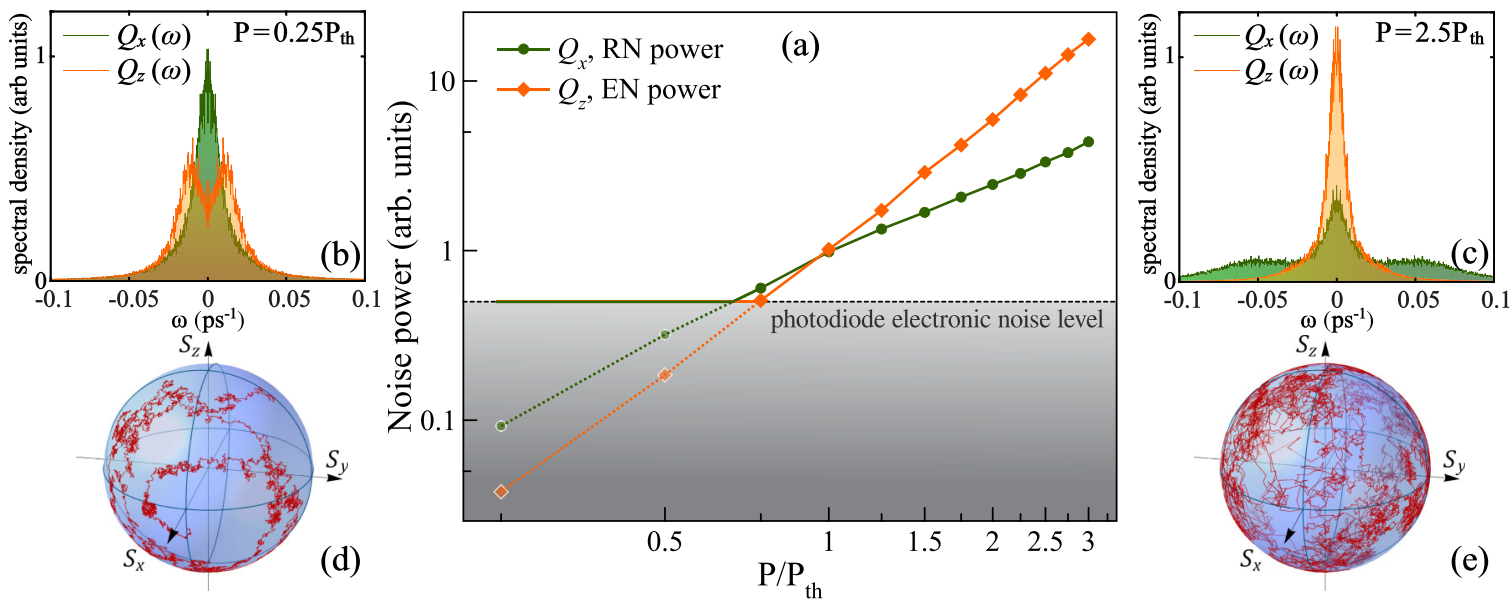

FIG. 3. Simulations of the noise dynamics of the condensate-emission polarization. The middle panel (a) shows the pump-power dependence of the zero-frequency spectral densities $Q_{x}(0)$ and $Q_{z}(0)$ correlated with the rotation and ellipticity noises, respectively. The dynamics was simulated with noisy initial conditions over $30 \mathrm{~ns}$, and the data were averaged over 100 realizations for each point. The dashed line denotes the approximate level of the photodiode electronic noise (shaded region) that limits the actual range of noise power measured in the experiment; see Fig. 2. The lower noise power values are indicated by the dotted parts of the curves. The stochastic behavior of the polariton condensate in the subthreshold regime at $P=0.25 P_{\text {th }}$ is illustrated in the left panels (b) and (d) while the right panels (c) and (e) correspond to the above-threshold pump power $P=2.5 P_{\text {th }}$. The full-range spectral densities $Q_{x}(\omega)$ and $Q_{z}(\omega)$ are shown in (b) and (c). The corresponding dynamics of the normalized condensate pseudospin $\mathbf{S} / S$ on the Poincaré sphere during $1.5 \mathrm{~ns}$ of evolution are shown in (d) and (e). The parameters used for simulations are $\alpha_{1}=10 \mu \mathrm{eV}, \alpha_{2}=-0.1 \alpha_{1}, \alpha_{r}=2 \alpha_{1}, R=0.01 \mathrm{ps}^{-1}, \gamma_{c}=0.01 \mathrm{ps}^{-1}, \gamma_{r}=2 \gamma_{c}, \delta_{l}=8 \mu \mathrm{eV}$.

where $P$ defines the pump intensity and $\gamma_{r}$ is a reservoir population relaxation parameter.

Note that for simplicity we neglect the spatial distributions of $\boldsymbol{\Psi}$ and $n$. This approach allows us to describe the typical behavior of the PE noise which is independent on the optical trap shape.

Equations (1) and (2) were solved numerically on timescales of several tens of nanoseconds; see Fig. 3. The polarization noise properties are governed by the behavior of the pseudospin (Stokes) vector of the condensate, whose components in the circular polarization basis read $S_{x}=\operatorname{Re}\left(\Psi_{-}^{*} \Psi_{+}\right)$, $S_{y}=\operatorname{Im}\left(\Psi_{-}^{*} \Psi_{+}\right), S_{z}=\frac{1}{2}\left(\left|\Psi_{+}\right|^{2}-\left|\Psi_{-}\right|^{2}\right)$. The stochastic dynamics predicts the width of the fluctuation spectrum to be comparable to the spectral line width, that is tenths of THz; see Figs. 3(b) and 3(c). The bandwidth of the experimentally used balanced photodiode was much smaller (limited by $1 \mathrm{MHz}$ ). Therefore the noise power measured with the balanced photoreceiver of the SNS scheme corresponds to the calculated spectral density $Q_{\beta}(\omega)=\int B_{\beta}(\tau) e^{-i \omega \tau} d \tau$ taken at zero frequency, $\omega=0$, that is $Q_{\beta}(0)=\int B_{\beta}(\tau) d \tau$. Here $B_{\beta}(\tau)=T^{-1} \int_{0}^{T} S_{\beta}(t) S_{\beta}(t+\tau) d t$ is a correlation function of the quantity $S_{\beta}$ with $\beta$ standing for the indices $(x, y, z)$ and $T$ being the time of calculations.

The calculated pump-intensity-dependence of the noise power, Fig. 3(c), agrees with the experimentally observed asymmetry between the subthreshold and the above-threshold regimes of the polarization noise behavior.

The observed asymmetry can be understood by examining the conservative dynamics of the Stokes vector, $\partial_{t} \boldsymbol{S} \propto-\boldsymbol{\Omega} \times$ $S$, which corresponds to its precession about the effective magnetic field $\boldsymbol{\Omega}=\left(\delta_{l}, 0,\left(\alpha_{1}-\alpha_{2}\right) S_{z}\right)$. The $x$ component of this field, $\Omega_{x}=\delta_{l}$, arises from the splitting of linear polar- izations and accounts for the rotation of the condensate pseudospin $S$ about $x$ axis. The field strength $\Omega_{x}$ does not depend on the pumping level. In contrast, the $z$-dependent effective field $\Omega_{z}=\left(\alpha_{1}-\alpha_{2}\right) S_{z}$ does depend on the pump and appears to be suppressed at the small condensate occupancies, $S_{z} \rightarrow 0$, typically below the threshold. An example of the fluctuation dynamics of the condensate pseudospin at the subthreshold pump is shown in Fig. 3(d). Due to the precession of the pseudospin $S_{y}$ and $S_{z}$ components, their zero-frequency noise power turns out to be suppressed, causing the RN power $Q_{x}(0)$ to exceed the EN power $Q_{z}(0)$; see Fig. 3(b) and [29].

An increase of the pump power above the threshold results in the growth of the condensate occupancy and the increase of the effective magnetic field generated due to spin-dependent polariton-polariton interactions. By virtue of the strong spin anisotropy of the polariton-polariton interactions, which are much stronger for the same spin than for the opposite spin polaritons $\left(\alpha_{1} \neq \alpha_{2}\right)$, the fluctuations of the population imbalance between the circularly polarized condensate components $S_{z}$ trigger the pseudospin rotation about $z$ axis. This effect is is a manifestation of the pseudospin self-induced Larmor precession [16].

Note that far above the threshold the fluctuations of the order parameter which are governed by the reservoir occupancy become so strong that the pseudospin vector spans all the Poincaré sphere; see Fig. 3(e). Therefore the degree of polarization of the condensate emission approaches zero, which is consistent with our experimental results.

Although the time-integrated value of $S_{z}$ is zero, the amplitude of its fluctuations grows with the pump power increase. Therefore, above the threshold the time-averaged modulus of $\Omega_{z}$ grows and at a certain pumping intensity, which is typically 
close to the threshold, becomes larger than $\Omega_{x}$. The effective magnetic field vector tilts to the $z$ axis. As a result the fast oscillations of the instant values of $S_{x}$ and $S_{y}$ components, being averaged over the time that essentially exceeds the inverse effective field strength, lead to suppression of $\mathrm{RN}$ power compared to the fluctuations of $S_{z}$; see Fig. 3(c).

This approach allows reproduction of the characteristic behavior which is qualitatively the same for any pump beam profile. An influence of the specific shape of the pump beam can be accounted for by rescaling of the reservoir to condensate scattering rate $R \rightarrow \beta R$. The factor $\beta<1$ accounts for the spatial overlap between the condensate and the reservoir, which is dependent on the pump beam profile and is expected to decrease with the growth of the optical trap size. Our simulations show that the variation of $\beta$ alters the position of the crossing point of the $\mathrm{EN}$ and $\mathrm{RN}$ pump power dependencies.

In conclusion, we show that the SNS technique is capable of revealing the intrinsic fluctuation dynamics of a spontaneously built-up exciton-polariton system even if the condensate emission is almost completely unpolarized. The behavior of the polarization noise, described in terms of the ellipticity noise and the polarization plane azimuth noise, demonstrates a strong asymmetry between the subthreshold and above-threshold regimes. This asymmetry stems from the competition of the linear polarization splitting and the effect of the self-induced Larmor precession of the condensate pseudospin. In particular, we found that below the polariton lasing threshold the fluctuation dynamics is superimposed by the pseudospin precession about the effective magnetic field oriented in the equatorial plane of the Poincaré sphere. We ascribe the appearance of this effective magnetic field to the microcavity mirror birefringence which arises from the mechanical strain caused by the lattice mismatch of the materials. Above the condensation threshold the effect of the self-induced Larmor precession builds up as the population of circular polarization states grows. These concurring effects lead to the intersection of the $\mathrm{EN}$ and $\mathrm{RN}$ pump-powerdependencies.

Acknowledgments. The work was financially supported by RFBR Grant No. 17-02-01112. The authors acknowledge Saint Petersburg State University for research support via Grant No. 51125686. The experiment was carried out at Saint Petersburg Resource Center "Nanophotonics." I.Y.C., P.G.S., and A.V.K. acknowledge support from Westlake University (Project No. 041020100118) and from Program 2018R01002 funded by the Leading Innovative and Entrepreneur Team Introduction Program of Zhejiang. A.T., Z.H., and P.G.S. are deeply thankful to the Russian Science Foundation for Grant No. 19-72-20120 for sample growth financial support. I.I.R. appreciates support for experimental work via RFBR Grant No. 19-52-12032.
[1] A. Imamoğlu, R. J. Ram, S. Pau, and Y. Yamamoto, Nonequilibrium condensates and lasers without inversion: Exciton-polariton lasers, Phys. Rev. A 53, 4250 (1996).

[2] A. Kavokin and G. Malpuech, in Cavity Polaritons, edited by V. M. Agranovich (Elsevier, Amsterdam, 2003).

[3] I. A. Shelykh, Yu. G. Rubo, G. Malpuech, D. D. Solnyshkov, and A. V. Kavokin, Polarization and Propagation of Polariton Condensates, Phys. Rev. Lett. 97, 066402 (2006).

[4] G. Christmann, R. Butté, E. Feltin, J. F. Carlin, and N. Grandjean, Room temperature polariton lasing in a GaN/AlGaN multiple quantum well microcavity, Appl. Phys. Lett. 93, 51102 (2008).

[5] Feng Li, L. Orosz, O. Kamoun, S. Bouchoule, C. Brimont, P. Disseix, T. Guillet, X. Lafosse, M. Leroux, J. Leymarie et al., From Excitonic to Photonic Polariton Condensate in a ZnO-Based Microcavity, Phys. Rev. Lett. 110, 196406 (2013).

[6] C. P. Dietrich, A. Steude, L. Tropf, M. Schubert, N. M. Kronenberg, K. Ostermann, S. Höfling, and M. C. Gather, An exciton-polariton laser based on biologically produced fluorescent protein, Sci. Adv. 2, e1600666 (2016).

[7] S. Pirotta, M. Patrini, M. Liscidini, M. Galli, G. Dacarro, G. Canazza, G. Guizzetti, D. Comoretto, and D. Bajoni, Strong coupling between excitons in organic semiconductors and Bloch surface waves, Appl. Phys. Lett. 104, 051111 (2014).

[8] T. Ellenbogen and K. B. Crozier, Exciton-polariton emission from organic semiconductor optical waveguides, Phys. Rev. B 84, 161304(R) (2011).

[9] D. Bajoni, P. Senellart, E. Wertz, I. Sagnes, A. Miard, A. Lemaître, and J. Bloch, Polariton Laser Using Single Micropil- lar GaAs-GaAlAs Semiconductor Cavities, Phys. Rev. Lett. 100, 047401 (2008).

[10] A. Das, P. Bhattacharya, J. Heo, A. Banerjee, and W. Guo, Polariton Bose-Einstein condensate at room temperature in an $\mathrm{Al}(\mathrm{Ga}) \mathrm{N}$ nanowire-dielectric microcavity with a spatial potential trap, Proc. Natl. Acad. Sci. USA 110, 2735 (2013).

[11] S. Christopoulos, G. Baldassarri, H. von Högersthal, A. J. D. Grundy, P. G. Lagoudakis, A. V. Kavokin, J. J. Baumberg, G. Christmann, R. Butté, E. Feltin, J.-F. Carlin, and N. Grandjean, Room-Temperature Polariton Lasing in Semiconductor Microcavities, Phys. Rev. Lett. 98, 126405 (2007).

[12] O. Jamadi, F. Reveret, P. Disseix, F. Medard, J. Leymarie, A. Moreau, D. Solnyshkov, C. Deparis, M. Leroux, E. Cambril et al., Edge-emitting polariton laser and amplifier based on a $\mathrm{ZnO}$ waveguide, Light Sci. Appl. 7, 82 (2018).

[13] E. Wertz, L. Ferrier, D. D. Solnyshkov, P. Senellart, D. Bajoni, A. Miard, A. Lemaître, G. Malpuech, and J. Bloch, Spontaneous formation of a polariton condensate in a planar GaAs microcavity, Appl. Phys. Lett. 95, 051108 (2009).

[14] P. Tsotsis, P. S. Eldridge, T. Gao, S. I. Tsintzos, Z. Hatzopoulos, and P. G. Savvidis, Lasing threshold doubling at the crossover from strong to weak coupling regime in GaAs microcavity, New J. Phys. 14, 023060 (2012).

[15] P. Bhattacharya, T. Frost, S. Deshpande, M. Z. Baten, A. Hazari, and A. Das, Room Temperature Electrically Injected Polariton Laser, Phys. Rev. Lett. 112, 236802 (2014).

[16] I. Shelykh, G. Malpuech, K. V. Kavokin, A. V. Kavokin, and P. Bigenwald, Spin dynamics of interacting exciton polaritons in microcavities, Phys. Rev. B 70, 115301 (2004). 
[17] F. P. Laussy, I. A. Shelykh, G. Malpuech, and A. V. Kavokin, Effects of Bose-Einstein condensation of exciton polaritons in microcavities on the polarization of emitted light, Phys. Rev. B 73, 035315 (2006).

[18] D. N. Krizhanovskii, D. Sanvitto, I. A. Shelykh, M. M. Glazov, G. Malpuech, D. D. Solnyshkov, A. Kavokin, S. Ceccarelli, M. S. Skolnick, and J. S. Roberts, Rotation of the plane of polarization of light in a semiconductor microcavity, Phys. Rev. B 73, 073303 (2006).

[19] D. Read, T. C. H. Liew, Y. G. Rubo, and A. V. Kavokin, Stochastic polarization formation in exciton-polariton BoseEinstein condensates, Phys. Rev. B 80, 195309 (2009).

[20] J. Levrat, R. Butteé, T. Christian, M. Glauser, E. Feltin, J.-F. Carlin, N. Grandjean, D. Read, A. V. Kavokin, and Y. G. Rubo, Pinning and Depinning of the Polarization of ExcitonPolariton Condensates at Room Temperature, Phys. Rev. Lett. 104, 166402 (2010).

[21] M. Müller, M. Oestreich, M. Römer, and J. Hübner, Semiconductor spin noise spectroscopy: Fundamentals, accomplishments, and challenges, Physica E 43, 569 (2010).

[22] V. S. Zapasskii, Spin-noise spectroscopy: From proof of principle to applications, Adv. Opt. Photon. 5, 131 (2013).

[23] J. Hübner, F. Berski, R. Dahbashi, and M. Oestreich, The rise of spin noise spectroscopy in semiconductors: From acoustic to GHz frequencies, Phys. Status Solidi B 251, 1824 (2014)
[24] I. I. Ryzhov, M. M. Glazov, A. V. Kavokin, G. G. Kozlov, M. Aßmann, P. Tsotsis, Z. Hatzopoulos, P. G. Savvidis, M. Bayer, and V. S. Zapasskii, Spin noise of a polariton laser, Phys. Rev. B 93, 241307(R) (2016).

[25] G. G. Kozlov, I. I. Ryzhov, A. Tzimis, Z. Hatzopoulos, P. G. Savvidis, A. V. Kavokin, M. Bayer, and V. S. Zapasskii, Hidden polarization of unpolarized light, Phys. Rev. A 98, 043810 (2018).

[26] I. A. Shelykh, K. V. Kavokin, A. V. Kavokin, G. Malpuech, P. Bigenwald, H. Deng, G. Weihs, and Y. Yamamoto, Semiconductor microcavity as a spin-dependent optoelectronic device, Phys. Rev. B 70, 035320 (2004).

[27] A. Askitopoulos, H. Ohadi, A. V. Kavokin, Z. Hatzopoulos, P. G. Savvidis, and P. G. Lagoudakis, Polariton condensation in an optically induced two-dimensional potential, Phys. Rev. B 88, 041308(R) (2013).

[28] A. Dreismann, H. Ohadi, Y. del-Valle Inclan Redondo, R. Balili, Y. G. Rubo, S. Tsintzos, G. Deligeorgis, Z. Hatzopoulos, P. G. Savvidis, and J. J. Baumberg, A sub-femtojoule electrical spinswitch based on optically trapped polariton condensates, Nat. Mater. 15, 1074 (2016).

[29] M. M. Glazov, M. A. Semina, E. Ya. Sherman, and A. V. Kavokin, Spin noise of exciton polaritons in microcavities, Phys. Rev. B 88, 041309(R) (2013). 